\title{
On Using Satisfiability-Based Pruning Techniques in Covering Algorithms
}

\author{
Vasco M. Manquinho \\ João Marques-Silva \\ vmm@algos.inesc.pt \\ jpms@inesc.pt \\ INESC/Cadence European Laboratories \\ Dept. of Informatics, Instituto Superior Técnico \\ Lisboa, Portugal
}

\begin{abstract}
Covering problems are widely used as a modeling tool in Electronic Design Automation (EDA). Recent years have seen dramatic improvements in algorithms for the Unate/Binate Covering Problem (UCP/BCP). Despite these improvements, $B C P$ is a well-known computationally hard problem, with many existing real-world instances that currently are hard or even impossible to solve. In this paper we apply search pruning techniques from the Boolean Satisfiability (SAT) domain to BCP. Furthermore, we generalize these techniques, in particular the ability to backtrack nonchronologically, to exploit the actual formulation of covering problems. Experimental results, obtained on representative instances of the Unate and Binate Covering Problems, indicate that the proposed techniques provide significant performance gains for different classes of instances.
\end{abstract}

\section{Introduction}

The Binate Covering Problem (BCP) finds many applications in Electronic Design Automation (EDA), examples of which include logic and sequential synthesis (state minimization and exact encoding), cell-library binding and minimization of Boolean relations [12]. In recent years, several powerful algorithmic techniques have been proposed for solving BCP, allowing dramatic improvements in the ability to solving large and complex instances of BCP. Examples of these techniques include, among others, partitioning [3], limit-lower bound [4], negative-thinking [8] (for unate covering), and linear-programming lower bounds [10]. Despite these improvements, and as with other NP-hard problems, new effective techniques allow in general very significant gains, both in the amount of search and in the run times. The ultimate consequence of proposing new algorithmic techniques is the potential ability for solving new classes of instances.

The main objective of this paper is to propose additional techniques for pruning the amount of search in branch-and-bound algorithms for solving covering problems. These techniques correspond to generalizations and extensions of similar techniques proposed in the Boolean Satisfiability (SAT) domain, where they have been shown to be highly effective [14]. In particular, and to our best knowledge, we provide for the first time conditions which enable branch-and-bound algorithms to backtrack non-chronologically whenever upper and lower bound conditions require bounding to take place.

This paper is organized as follows. In Section 2 the notation used throughout the paper is introduced. Afterwards, branch-and-bound covering algorithms are briefly reviewed, giving emphasis to solutions based on SAT algorithms. In Section 4 we propose new techniques for reducing the amount of search. In particular we show how effective search pruning techniques from the SAT domain can be generalized and extended to the BCP domain. Experimental results are presented in Section 5, and the paper concludes in Section 6.

\section{Preliminaries}

An instance $C$ of a covering problem is defined as follows,

$$
\begin{array}{ll}
\operatorname{minimize} & \sum_{j=1}^{n} c_{j} \cdot x_{j} \\
\text { subject to } & A \cdot x \geq b, \quad x \in\{0,1\}^{n}
\end{array}
$$

where $c_{j}$ is a non-negative integer cost associated with variable $x_{j}, 1 \leq j \leq n$ and $A \cdot x \geq b, x \in\{0,1\}^{n}$ denote the set of linear constraints. If every entry $(m \times n)$ of matrix $A$ is in the set $\{0,1\}$ and $b_{i}=1,1 \leq i \leq m$, then $C$ is an instance of the unate covering problem (UCP). Moreover, if the entries $a_{i j}$ of $A$ belong to $\{-1,0,1\}$ and $b_{i}=1-\left|\left\{a_{i j}: a_{i j}=-1\right\}\right|$, then $C$ is an instance of the binate covering problem (BCP). It is interesting to observe that if $C$ is an instance of the binate covering problem, then each constraint can be interpreted as a propositional clause.

Conjunctive Normal Form (CNF) formulas are introduced next. Because the set of constraints of an instance $C$ of BCP is equivalent to a CNF formula, and also because some of the search pruning techniques described in the remainder of the paper are easier to convey in this alternative representation. 
A propositional formula $\varphi$ in Conjunctive Normal Form (CNF) denotes a boolean function $f:\{0,1\}^{n} \rightarrow\{0,1\}$. The formula $\varphi$ consists of a conjunction of propositional clauses, where each clause $\omega$ is a disjunction of literals, and a literal $l$ is either a variable $x_{j}$ or its complement $\bar{x}_{j}$. If a literal assumes value 1 , then the clause is satisfied. If all literals of a clause assume value 0 , the clause is unsatisfied. Clauses with only one unassigned literal are referred to as unit. Finally, clauses with more than one unassigned literal are said to be unresolved. In a search procedure, a conflict is said to be identified when at least one clause is unsatisfied. We should also observe that a clause $\omega=\left(l_{1}+\cdots+l_{k}\right), k \leq n$, can be interpreted as a linear inequality $l_{1}+\cdots+l_{k} \geq 1$, and the complement of a variable $x_{j}, \bar{x}_{j}$, can be represented by $1-x_{j}$.

When a clause is unit (with only one unassigned literal) an assignment can be implied. For instance, consider a propositional formula $\varphi$ which contains clause $\omega=\left(x_{1}+\right.$ $\bar{x}_{2}$ ) and assume that $x_{2}=1$. For $\varphi$ to be satisfied, $x_{1}$ must be assigned value 1 due to $\omega$. Therefore, we say that $x_{2}=1$ implies $x_{1}=1$ due to $\omega$ or that clause $\omega$ explains the assignment $x_{1}=1$. These logical implications correspond to the application of the unit clause rule [5] and the process of repeatedly applying this rule is called boolean constraint propagation [14]. We should note that throughout the remainder of this paper some familiarity with backtrack search SAT algorithms is assumed. The interested reader is referred to the bibliography (see for example $[1,14]$ for additional references).

Covering problems are often solved by branch and bound algorithms $[3,8,15]$. In these cases, each node of the search tree corresponds to a selected unassigned variable and the two branches out of the node represent the assignment of 1 and 0 to that variable. These variables are named decision variables. The first node is called the root (or the top node) of the search tree and corresponds to the first decision level. Hence, the top nodes define the first decision levels of the search tree.

\section{Backtrack Search Algorithms for Covering Problems}

The most widely known approach for solving covering problems is the classical branch and bound procedure [9], in which upper bounds on the value of the cost function are identified and lower bounds of the cost function are estimated considering the current set of variable assignments. The search can be pruned whenever the lower bound estimation is higher than or equal to the current upper bound. In these cases we can guarantee that a better (lower cost) solution cannot be found with the current variable assignments and therefore the search can be pruned. The algorithms described in $[3,10,15]$ follow this approach.

There are several lower bound estimation procedures that can be used, namely the ones based on linear-programming relaxations [10] or lagrangian relaxations [13], but the approximation of a maximum independent set of clauses [4] is the most common one. The tightness of the lower bounding procedure is crucial for the algorithm's efficiency, because with higher estimates of the lower bound, the search can be pruned sooner. For a better understanding, a method of approximation of a maximum independent set of clauses is described in section 3.3.

Covering algorithms also incorporate several powerful reduction techniques such as clause and variable dominance, row consensus, Gimpel's reduction [7], the limit lower bound theorem [4] and partitions [3]. A comprehensive overview of these methods can be found in $[2,15]$.

In the next few sections we briefly review alternative approaches for solving $\mathrm{BCP}$, which are known to be competitive for specific types of instances, e.g. when the constraints are hard to solve. These approaches, namely the ones based on boolean satisfiability algorithms, have different pruning strategies which are not commonly used in branch and bound algorithms for solving BCP. In section 3.2 an algorithm which combines features from both approaches is described.

\subsection{SAT-Based Linear Search Algorithm}

In [1] P. Barth describes how to solve pseudo-boolean optimization (i.e. a generalization of BCP) using a propositional satisfiability (SAT) algorithm. However, the algorithm described in [1] is based on the Davis-Putnam [5] procedure, which has been shown to be particularly inefficient for a large number of instances of SAT. In [11], a new algorithm based on the GRASP SAT algorithm [14] is proposed, which is able to obtain better experimental results. Both these two algorithms interpret the binate covering problem (BCP) as a SAT problem defined by the constraints $A \cdot x \geq b$, but with the additional constraint of having to find a solution with cost lower than an existing upper bound value. The possible values assumed by the cost function for the different assignments to the problem variables $\left\{x_{1}, \ldots, x_{n}\right\}$ range from 0 , when all variables are assigned value 0 , to $\sum_{j=1}^{n} c_{j}$, when all variables with $c_{j}>0$ are assigned value 1 . Initially, the upper bound $u b$ on the value of the cost function is given by:

$$
u b=\sum_{j=1}^{n} c_{j}+1
$$

SAT-based linear search algorithms perform a linear search on the possible values of the cost function, starting from the highest possible value. Whenever a new solution is found that satisfies all the constraints, the upper bound $u b$ is updated to:

$$
u b=\sum_{j=1}^{n} c_{j} \cdot x_{j}
$$




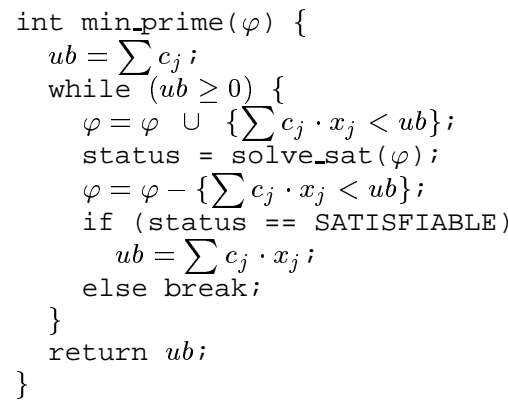

Figure 1. SAT-based linear search algorithm

If the resulting SAT problem is not satisfiable, then the solution to the BCP problem is given by $u b$. Starting with the $u b$ given by (2), SAT-based linear search algorithms consist on the application of the following steps:

1. Create a new constraint $\sum_{j=1}^{n} c_{j} \cdot x_{j}<u b$. This inequality basically requires that a computed solution must have a cost lower than the best one found so far.

2. Solve the resulting instance of a satisfiability problem, defined on linear inequalities. The modification of most SAT algorithms to deal with this generalization is straightforward.

3. If the instance is satisfiable, then update $u b$ according to (3) and go back to step 1. Otherwise, the solution to the covering problem is $u b$. In those cases where the initial upper bound is never updated, the problem does not have a solution.

\subsection{SAT-Based Branch and Bound Algorithm}

Additional SAT-based BCP algorithms have been proposed. In [11] a new algorithmic organization is described, consisting in the integration of several features from SAT algorithms in a branch and bound procedure, bsolo, to solve the binate covering problem. This new framework from bsolo incorporates the main features from both approaches, namely the bounding procedure and reduction techniques from branch and bound algorithms, and search pruning techniques from SAT algorithms.

Originally, the algorithm presented in [11] already incorporated the main pruning techniques of the GRASP SAT algorithm [14]. To our knowledge, bsolo was the first branch and bound algorithm for solving BCP that implemented a non-chronological backtracking search strategy, clause recording and identification of necessary assignments. Mainly due to an effective conflict analysis procedure which allows non-chronological backtracking steps to be identified, bsolo performs better than other branch and bound algorithms in several classes of instances, as shown

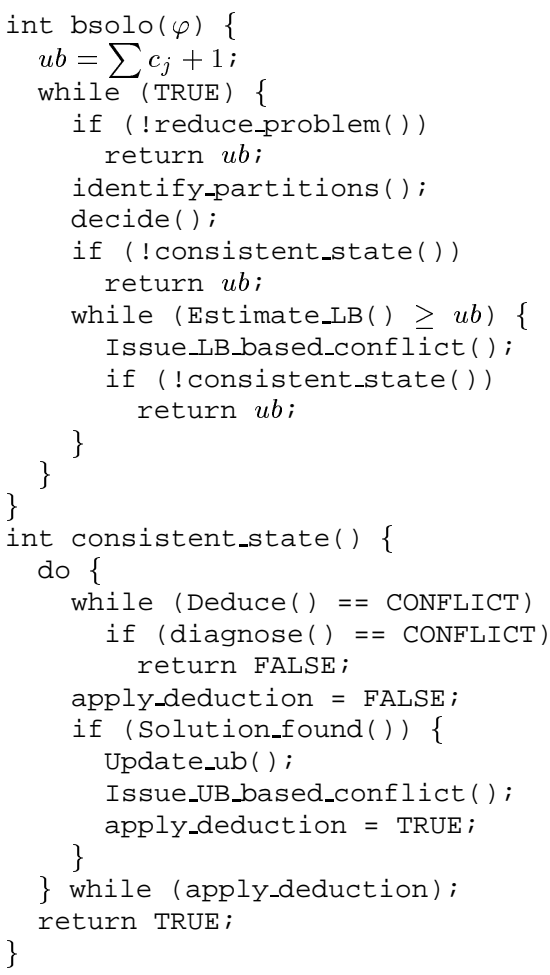

\section{Figure 2. SAT-based branch and bound algo- rithm}

in [11]. However, non-chronological backtracking was limited to just one specific type of conflict. In section 4 we describe an extension which allows non-chronological backtracking for all types of conflicts. The main steps of the algorithm (fig. 2) can be described as:

1. Initialize the upper bound to the highest possible value as defined in (2).

2. Apply function reduce problem to reduce the problem dimension by applying the techniques from standard branch and bound covering algorithms. Afterwards, identify problem partitions and branch on a given decision variable (i.e. make a decision assignment).

3. The function consistent_state verifies whether the current state doesn't have any conflicts. This is done by applying boolean constraint propagation and if a conflict is reached, apply the conflict analysis procedure, record relevant clauses and proceed with the search procedure or backtrack if necessary.

4. If a solution to the constraints has been identified, update the upper bound according to (3) and issue an $u p$ per bound conflict to backtrack on the search tree.

5. Estimate a lower bound given the current variable assignments. If this value is higher than or equal to the 
current upper bound, issue a lower bound conflict and bound the search by applying the conflict analysis procedure to determine the node to backtrack to (using function consistent_state). Continue the search from step 2 .

\subsection{Maximum Independent Set of Clauses}

The estimation of lower bounds on the value of the cost function is a very effective method to prune the search tree and the accuracy of the used procedure is critical to identify useless areas of the search space. This section describes a greedy method to estimate a lower bound based on independent set of clauses that is outlined for example in [4].

This procedure consists in finding a set $I$ of disjoint unate clauses, i.e., with no literals in common between them. Since maximizing the cost of $I$ is a NP-hard problem, a greedy computation is used like the one described in fig. 3. The effectiveness of this method largely depends on the clauses added to $I$. Usually, it is chosen the clause which maximizes the ratio between its weight and its number of elements.

The minimum cost to satisfy $I$ is a lower bound of the problem and is given by

$$
\begin{aligned}
& \operatorname{Cost}(I)=\sum_{\omega \in I} W \operatorname{eight}(\omega) \quad \text { where } \\
& W \operatorname{eight}(\omega)=\min _{x_{j} \in \omega} c_{j}
\end{aligned}
$$

\subsection{Upper and Lower Bound Conflicts}

From section 3.2 we know that in bsolo there are three types of conflicts which can arise: logical conflicts that occur when one of the problem instance constraints is unsatisfiable, upper bound conflicts that occur when a solution to the constraints is found, and lower bound conflicts that take place when the lower bound is higher than or equal to the upper bound. When logical conflicts occur, the conflict analysis procedure from GRASP is applied and determines to which decision level the search should backtrack to (possibly in a non-chronological manner).

However, the other two types of conflicts are treated differently. In bsolo, whenever we have an upper or lower bound conflict, a new clause must be added to the problem instance in order for a logical conflict to be issued and, consequently, to bound the search. This requirement is inherited from the GRASP SAT algorithm where, for guaranteeing completeness, both conflicts and implied variable assignments must be explained in terms of the existing variable assignments [14]. With respect to conflicts, each recorded conflict clause is built using the assignments that are deemed responsible for the conflict to arise. If the assignment $x_{j}=1$ (or $x_{j}=0$ ) is considered responsible, the literal $\bar{x}_{j}$ (respectively, literal $x_{j}$ ) is added to the conflict

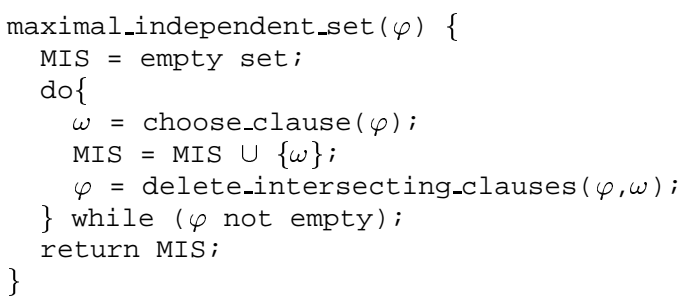

Figure 3. Algorithm for computing a MIS

clause. This literal basically states that in order to avoid the conflict one possibility is certainly to have the assignment $x_{j}=0$ (respectively, $x_{j}=1$ ). Clearly, by construction, after the clause is built its state is unsatisfied. Consequently, the conflict analysis procedure has to be called to determine to which decision level the algorithm must backtrack to. Hence the search is bound.

We start by studying upper bound conflicts. In these situations, one possible approach to build a clause to bound the search would be to include all decision variables in the search tree. In this case, the conflict would always depend on the last decision variable. Therefore, backtracking due to upper bound conflicts would necessarily be chronological (i.e. to the previous decision level), hence guaranteeing that the algorithm would be complete.

The previous strategy can also be used for lower bound conflicts. By building a clause involving all decision assignments present in the search tree, we guarantee that the search is bound and ensure that the algorithm is complete. Suppose that the set $\left\{x_{1}=1, x_{2}=0, x_{3}=0, x_{4}=1\right\}$ corresponds to those decisions and $\omega_{l b}$ is the clause to be added. Then we would have $\omega_{l b}=\left(\bar{x}_{1}+x_{2}+x_{3}+\bar{x}_{4}\right)$. Again, the problem with this approach (which was used in [11] for the original bsolo algorithm) is that backtracking is always chronological, since it depends on all decisions made. In sections 4.1 and 4.2 we will present new ways for building these clauses, which enable non-chronological backtracking due to upper and lower bound conflicts.

\section{SAT-Based Pruning Techniques for BCP}

One of the main features of bsolo is the ability to backtrack non-chronologically when conflicts arise. This feature is enabled by the conflict analysis procedure inherited from the GRASP SAT algorithm. However, as illustrated in section 3.4, in the original bsolo algorithm non-chronological backtracking was only possible for logical conflicts. In the case of an upper or lower bound conflict all the search tree decision assignments were used to explain the conflict. Therefore, these conflicts would always depend on the most recent decision assignment and backtracking would always be chronological.

In this section we will show that it is possible to compute sets of assignments which are responsible for upper and lower bound conflicts. Moreover, since the assignments 
that explain each conflict can be from earlier decision levels in the search tree, non-chronological backtracking can take place.

\subsection{Dependencies in Upper Bound Conflicts}

As mentioned in Section 3.4, upper bound conflicts correspond to the process of bounding the search when a new solution (with lower cost) is found. In bsolo, and because of the conflict analysis procedure, the bounding process requires creating a new conflict clause. Moreover, in the original version of bsolo all decision variables were present in this clause, thus preventing non-chronological backtracks from occurring. However, it is straightforward to conclude that the assignments which characterize the computed solution are the ones that allow the value of the cost function to grow, i.e., the assignments of 1 to variables with positive cost in the cost function. Therefore, we should backtrack to a decision level where at least one of these assignments is toggled to its complemented value. Let $\omega_{u b}$ be the clause added due to an upper bound conflict. This clause is defined by:

$$
\omega_{u b}=\left\{l=\bar{x}_{j}: \operatorname{Cost}\left(x_{j}\right)>0 \wedge x_{j}=1\right\}
$$

Consequently, it becomes possible to backtrack nonchronologically after identifying an upper bound conflict. This is illustrated next.

Let $f\left(x_{1}, x_{2}, x_{3}, x_{4}\right)=x_{1}+x_{2}+x_{3}$ be the cost function to minimize, and the set of constraints be:

$$
\left(x_{1}+x_{4}\right) \cdot\left(\bar{x}_{4}+\bar{x}_{2}\right) \cdot\left(x_{3}+x_{4}\right) \cdot\left(\bar{x}_{3}+x_{4}\right)
$$

Let us assume the sequence of decision assignments $x_{1}=1$ and $x_{2}=0$. Suppose that the next decision assignment is $x_{3}=1$, that implies $x_{4}=1$. Then all clauses are satisfied, and the value of the cost function is 2 . Next, an upper bound conflict is issued, and the clause $\left(\bar{x}_{1}+\bar{x}_{3}\right)$ is created (observe that the assignment $x_{2}=0$ is irrelevant for being able to reduce the current upper bound estimate). Afterwards, the assignment $x_{3}=0$ is implied, which again implies $x_{4}=1$, thus satisfying all clauses. In this case the value of the cost function is 1 . Now, since the value of $x_{3}$ is implied, we can readily create the clause $\left(\bar{x}_{1}\right)$, which indicates that we should backtrack immediately to the decision stage where the assignment $x_{1}=1$ is defined. Hence, we backtrack non-chronologically, skipping the backtrack to the decision assignment $x_{2}=0$.

\subsection{Dependencies in Lower Bound Conflicts}

A lower bound conflict in a binate covering problem (BCP) $C$ arises when the lower bound is equal to or higher than the upper bound and we can write this condition as follows:

$$
\text { C.path }+ \text { C.lower } \geq \text { C.upper }
$$

where C.path is the cost of the assignments already made, C.lower is a lower bound estimate on the cost for satisfying the clauses not yet satisfied, and C.upper is the best (lowest cost) solution found so far. From the previous equation, we can readily conclude that $C$.path and $C$.lower are the unique components involved in each lower bound conflict. (Notice that C.upper is just the value of the cost function for a solution computed earlier in the search process.) Therefore, we will analyze both components in order to establish the assignments responsible for a given lower bound conflict.

We start by studying C.path. Clearly, the variable assignments that cause the value of $C$.path to grow are solely those assignments with a value of 1 . Hence, we can define a set of literals $\omega_{c p}$, such that each variable in $\omega_{c p}$ has positive cost and is assigned value 1 :

$$
\omega_{c p}=\left\{l=\bar{x}_{j}: \operatorname{Cost}\left(x_{j}\right)>0 \wedge x_{j}=1\right\}
$$

which basically states that to decrease the value of the cost function (i.e. C.path) at least one variable that is assigned value 1 has instead to be assigned value 0 .

We now consider C.lower. Let MIS be the independent set of clauses, obtained by the method described in section 3.3, that determines the value of C.lower. Note that each clause in MIS is part of MIS because it is neither satisfied nor covered by some other clause in MIS. Clearly, for each clause $w_{i}$ these conditions only hold due to the literals in $w_{i}$ that are assigned value 0 . If any of these literals was assigned value $1, w_{i}$ would certainly not be in MIS. Consequently, we can define a set of literals that explain the value of C.lower:

$$
\omega_{c l}=\left\{l: l=0 \wedge l \in \omega_{i} \wedge \omega_{i} \in M I S\right\}
$$

Now, as stated above, a lower bound conflict is solely due to the two components C.path and C.lower. Hence, this lower bound conflict will hold as long as the following clause $\omega_{l b}$ is unsatisfied:

$$
\omega_{l b}=\omega_{c p} \cup \omega_{c l}
$$

As long as this clause is unsatisfied, the values of C.path and C.lower will remain unchanged, and so the lower bound conflict exists. We can thus use this unsatisfied clause $\omega_{l b}$ to analyze the lower bound conflict and decide where to backtrack to, using the conflict analysis procedure of GRASP [14]. We should observe that backtracking can be non-chronological, because clause $\omega_{l b}$ does not necessarily depend on all decision assignments.

With respect to (11) a more careful analysis allows us to conclude that not all of the literals from $\omega_{c p}$ are necessary. Suppose that the lower bound is higher than the upper bound and define this difference as diff $=$ C.path + C.lower C.upper. It is possible to remove some literals from $\omega_{c p}$ such that the sum of the cost of the corresponding assignments is lower than diff. This is possible due to the fact that the conflict will still hold no matter the value of these 
assignments. For implementing this technique one interesting problem is to decide which literals should be removed. In bsolo an heuristic procedure is used for removing the literals that have been assigned at the most recent levels of the decision tree. Consequently, the likelihood of backtracking non-chronologically is higher, since these conflicts will be more dependent on the earlier levels of the search tree.

It is interesting to observe that a clause resulting from a lower bound conflict can be simpler. We have only described how simplifications can be made to the C.path component $\left(\omega_{c p}\right)$, but other simplifications can also be applied to the literals added due to the independent set of clauses (MIS) $\left(\omega_{c l}\right)$. Suppose we have a literal $l=x_{j}$, with $x_{j} \in \omega_{c l}$ and let $x_{j}=0$. If $x_{j}$ only belongs to one clause $\omega_{i}$ of the independent set and its cost is higher than or equal to the minimum cost of $\omega_{i}$, then $l$ can be removed from $\omega_{l b}$. To better understand how this is possible, suppose that $x_{j}=1$. In this situation, $\omega_{i}$ would not be in the independent set (it would be a satisfied clause) and the C.lower component would be lower ${ }^{1}$. However, since the cost of the variable is higher than or equal to the minimum cost of $\omega_{i}$, the $C$.path component would be higher, and hence the conflict would still hold. So, the assignment $x_{j}=0$ is irrelevant for the conflict to arise and $l$ can be removed from $\omega_{l b}$.

\subsection{Handling Reduction Techniques}

As mentioned in the previous sections, for implementing non-chronological backtracking each implied variable assignment needs to be properly explained, in order to guarantee that the resulting branch-and-bound algorithm is complete. Consequently, it is necessary that, whenever there is a variable assignment implied due to the application of a reduction technique (e.g., variable dominance, limit lower bound theorem, etc.), a new clause is built and added to the problem instance as an explanation for that assignment. Clearly, we could create this new clause by using all decision assignments in the decision tree, but this would negatively affect the ability of the search algorithm to backtrack non-chronologically. As before, we must identify conditions for using a reduced set of assignments instead of all decision assignments. In this section we illustrate how this is done for assignments implied due to the application of the limit lower bound theorem. For the other reduction techniques, a similar approach can be used.

The limit lower bound theorem [3] is applied to a variable $x_{j}$ whenever,

$$
\text { C.upper }-(\text { C.path }+ \text { C.lower }) \leq \operatorname{Cost}\left(x_{j}\right)
$$

In these cases, the assignment $x_{j}=0$ is implied.

\footnotetext{
${ }^{1}$ In fact, if the C.lower would be recomputed all over again, it is not guaranteed that it would decrease. Nevertheless, we know that without clause $\omega$ satisfied by $x_{j}=1, M I S \backslash\{\omega\}$ it is still an independent set of clauses. Therefore, $M I S \backslash\{\omega\}$ can be used as a low estimation of C.lower.
}

\begin{tabular}{|r|r|r|r|r|r|}
\hline & \multicolumn{2}{|c|}{ bsolo } & \multicolumn{2}{c|}{ scherzo } \\
\hline Benchmark & min. & CPU & Dec. & CPU & Dec. \\
\hline 5xp1.b & 12 & 181.02 & 1640 & 4.5 & 2234 \\
\hline 9sym.b & 5 & 27.91 & 135 & 3.6 & 320 \\
\hline alu4.b & - & ub 51 & time & - & time \\
\hline apex4.a & 776 & ub 781 & time & 87.4 & 48359 \\
\hline bench1.pi & - & ub 123 & time & - & time \\
\hline clip.b & 15 & 67.09 & 1313 & 0.6 & 97 \\
\hline count.b & 24 & 12.27 & 102 & 478.0 & 299780 \\
\hline e64.b & - & ub 48 & time & - & mem. \\
\hline ex5.pi & - & ub 68 & time & - & time \\
\hline exam.pi & - & ub 64 & time & - & time \\
\hline f51m.b & 18 & 97.00 & 1671 & 1.9 & 1586 \\
\hline jac3 & 15 & ub 17 & time & 4.9 & 292 \\
\hline max1024.pi & - & ub 262 & time & - & time \\
\hline prom2.pi & - & ub 297 & time & - & time \\
\hline rot.b & - & ub 120 & time & - & time \\
\hline sao2.b & 25 & 9.58 & 281 & 0.9 & 279 \\
\hline test4.pi & - & ub 102 & time & - & time \\
\hline
\end{tabular}

Table 1. Results for bsolo and scherzo

Let $\omega_{l l b}$ be a clause that must be added in order to explain the assignment $x_{j}=0$, which is implied by applying the limit lower bound theorem. Notice that this theorem is applied because of the values of C.path and C.lower. Thus, the assignments that explain these two values are also the explanation sought for the assignment $x_{j}=0$. Therefore, clause $\omega_{l l b}$ is constructed as follows,

$$
\omega_{l l b}=\omega_{c p} \cup \omega_{c l} \cup\left\{\bar{x}_{j}\right\}
$$

where $\omega_{c p}$ and $\omega_{c l}$ are the literals which explain the values in C.path and C.lower, as described in section 4.2. Therefore, $\omega_{l l b}$ becomes a new unit clause and consequently implies the assignment $x_{j}=0$. (Hence, we say that the assignment $x_{j}=0$ is explained by $\omega_{l l b}$.)

\section{Experimental Results}

In this section we include experimental results of several algorithms in two different sets of benchmarks. The first two tables have results from the MCNC benchmark set [16], while the others are from minimum-size test pattern problems [6]. All execution times are from a SUN Sparc Ultra I, running at $170 \mathrm{MHz}$, and with $100 \mathrm{MB}$ of available physical memory. The run time of every experiment was limited to 1 hour.

Whenever an algorithm was not able to find the optimum value for a given problem instance, the best computed upper bound is shown (provided the algorithm was able to compute one). In some situations, the reason for the algorithm to abort is shown. This can be because the time limit was reached or because the available memory was not enough.

In table 1 we present a comparison between bsolo and scherzo in the MCNC benchmark set. scherzo [3] is a classical branch and bound algorithm with powerful problem reduction techniques and very effective in this set of benchmarks, since most clauses only have positive literals. 


\begin{tabular}{|r|r|r|r|r|r|}
\hline \multicolumn{2}{|c|}{ bsolo } & \multicolumn{4}{|c|}{ no LB explanation } \\
\hline Benchmark & min. & CPU & Dec. & NCB & Jump \\
\hline cordic_Fa2@0 & 6 & 0.14 & 48 & 14 & 5 \\
\hline cordic_Fa2@1 & 6 & 0.25 & 94 & 6 & 3 \\
\hline cordic_Fa3@0 & 6 & 0.16 & 53 & 14 & 5 \\
\hline cordic_Fa3@1 & 6 & 0.25 & 100 & 6 & 4 \\
\hline cordic_Fa4@1 & 6 & 0.17 & 84 & 4 & 3 \\
\hline cordic_Fa6@0 & 6 & 0.17 & 58 & 8 & 3 \\
\hline misex1_Fd0@1 & 4 & 0.36 & 39 & 0 & 1 \\
\hline misex1_Fd1@0 & 4 & 0.32 & 53 & 3 & 5 \\
\hline misex1_Fd2@0 & 3 & 0.28 & 39 & 3 & 4 \\
\hline misex1_Fd3@1 & 3 & 0.36 & 48 & 5 & 4 \\
\hline misex1_Fy@0 & 5 & 0.04 & 12 & 1 & 3 \\
\hline misex1_Fy@1 & 5 & 0.04 & 10 & 1 & 2 \\
\hline misex3_Fa@0 & 9 & 112.60 & 1352 & 34 & 7 \\
\hline misex3_Fa@1 & 9 & 42.09 & 756 & 25 & 5 \\
\hline misex3_Fb@0 & 9 & 313.87 & 1887 & 24 & 6 \\
\hline misex3_Fb@1 & 8 & 96.27 & 1078 & 26 & 6 \\
\hline pcler8_Fi@0 & 2 & 0.26 & 40 & 14 & 2 \\
\hline pcler8_Fi@1 & 2 & 0.41 & 78 & 9 & 2 \\
\hline pcler8_Fj@1 & 4 & 0.21 & 87 & 11 & 2 \\
\hline pcler8_Fk@1 & 4 & 0.53 & 119 & 3 & 2 \\
\hline term1_Fa@0 & 4 & 0.12 & 56 & 1 & 2 \\
\hline term1_Fb@0 & 7 & 0.37 & 125 & 10 & 3 \\
\hline term1_Fb@1 & 7 & 0.31 & 140 & 13 & 3 \\
\hline term1_Fc@0 & 4 & 0.30 & 77 & 9 & 4 \\
\hline term1_Fd@1 & 4 & 0.39 & 92 & 10 & 9 \\
\hline
\end{tabular}

Table 2. Not using lower bound explanations

Clearly, scherzo is able to solve more problems and is faster in most problems. In this benchmark set, the main features of bsolo are not extensively used. We note however that there are some problems in which fewer decisions are made.

We should note that the bookkeeping required for the correct implementation of the SAT-based techniques can introduce noticeable computational overhead in bsolo. For the above instances, the gain obtained from the SAT-based techniques is low since non-chronological backtracking is almost non-existing, suggesting that further work must be done towards reducing the computed sets of dependencies.

As noted earlier, SAT-based BCP algorithms are better suited for instances whose constraints are hard to satisfy. In tables 2 and 3 we present the results of bsolo for benchmarks from minimum-size test pattern problems [6]. For these tables, and besides the CPU time and the number of decisions, the number of non-chronological backtracks and the highest jump made in the search tree are also included. In the first table, bsolo does not use the lower bound explanation described in section 4 and the non-chronological backtracks are just due to logical or upper bound conflicts. In table 3 the lower bound explanation of section 4 is used and we can see that bsolo is able to increase the number of non-chronological backtracks while significantly reducing the amount of search and the execution time for most instances.

Finally, in tables 4 and 5 we present a comparison between several algorithms for this set of instances. Table 4 clearly shows that general purpose algorithms for solving 01-Integer Linear Programs (lp-solve and cplex) perform

\begin{tabular}{|c|c|c|c|c|c|}
\hline \multicolumn{2}{|l|}{ bsolo } & \multicolumn{4}{|c|}{ LB explanation } \\
\hline Benchmark & min. & $\mathrm{CPU}$ & Dec. & $\mathrm{NCB}$ & Jump \\
\hline cordic_Fa2@0 & 6 & 0.21 & 47 & 14 & 4 \\
\hline cordic_Fa2@1 & 6 & 0.24 & 99 & 6 & 3 \\
\hline cordic_Fa3@0 & 6 & 0.18 & 52 & 14 & 4 \\
\hline cordic_Fa3@1 & 6 & 0.27 & 105 & 6 & 4 \\
\hline cordic_Fa4@1 & 6 & 0.18 & 84 & 4 & 3 \\
\hline cordic_Fa6@0 & 6 & 0.20 & 58 & 8 & 3 \\
\hline misex1_Fd0@1 & 4 & 0.25 & 25 & 0 & 1 \\
\hline misex1_Fd1@0 & 4 & 0.18 & 38 & 4 & 5 \\
\hline misex1_Fd2@0 & 3 & 0.23 & 35 & 3 & 4 \\
\hline misex1_Fd3@1 & 3 & 0.28 & 39 & 4 & 4 \\
\hline misex1_Fy@0 & 5 & 0.03 & 12 & 1 & 3 \\
\hline misex1_Fy@1 & 5 & 0.04 & 10 & 1 & 2 \\
\hline misex3_Fa@0 & 9 & 52.02 & 834 & 78 & 14 \\
\hline misex3_Fa@1 & 9 & 30.58 & 642 & 56 & 9 \\
\hline misex3_Fb@0 & 9 & 95.83 & 1152 & 119 & 9 \\
\hline misex3_Fb@1 & 8 & 79.69 & 978 & 70 & 8 \\
\hline pcler8_Fi@0 & 2 & 0.31 & 40 & 14 & 2 \\
\hline pcler8_Fi@1 & 2 & 0.39 & 78 & 9 & 2 \\
\hline pcler8_Fj@1 & 4 & 0.21 & 87 & 11 & 2 \\
\hline pcler8_Fk@1 & 4 & 0.51 & 121 & 4 & 2 \\
\hline term1_Fa@0 & 4 & 0.12 & 56 & 1 & 2 \\
\hline term1_Fb@0 & 7 & 0.31 & 90 & 6 & 3 \\
\hline term1_Fb@1 & 7 & 0.27 & 100 & 7 & 3 \\
\hline term1_Fc@0 & 4 & 0.29 & 74 & 9 & 4 \\
\hline term1_Fd@1 & 4 & 0.32 & 85 & 10 & 8 \\
\hline
\end{tabular}

Table 3. Using lower bound explanations

poorly. The same is true for scherzo which is not able to apply its main features in solving these instances. In table 5 we present the results for the SAT-based algorithms. The SAT-based linear search algorithms opbdp [1] and minprime [11] are able to solve all benchmarks. Moreover, bsolo results are significantly better than the results of both opbdp and min-prime, mainly due to the new techniques proposed in this paper.

\section{Conclusions}

This paper extends well-known search pruning techniques, from the Boolean Satisfiability domain, to branchand-bound algorithms for solving Unate and Binate Covering Problems. Besides detailing a branch-and-bound BCP algorithm built on top of a SAT solver, the paper describes conditions that allow for non-chronological backtracking in the presence of upper and lower bound conflicts. In addition, the paper also describes how reduction techniques, commonly used in BCP solvers, can be re-defined and utilized within a conflict analysis procedure, in such a way that non-chronological backtracking is enabled. To our best knowledge, this is the first time that branch-and-bound algorithms are augmented with the ability for backtracking non-chronologically in the presence of conflicts that result from upper and lower bound conditions.

Preliminary results obtained on several instances of the Unate and Binate Covering problems indicate that the proposed techniques are indeed effective and can be of crucial significance for specific classes of instances. 


\begin{tabular}{|r|r|r|r|r|}
\hline \multicolumn{2}{|c|}{} & lp-solve & scherzo & cplex \\
\hline Benchmark & min. & CPU & CPU & CPU \\
\hline cordic_Fa2@0 & 6 & 200.3 & 64.02 & 2.77 \\
\hline cordic_Fa2@1 & 6 & time & 94.90 & 12.22 \\
\hline cordic_Fa3@0 & 6 & 969.5 & 67.84 & 2.20 \\
\hline cordic_Fa3@1 & 6 & ub 7 & 97.37 & 9.02 \\
\hline cordic_Fa4@1 & 6 & time & 84.13 & 3.12 \\
\hline cordic_Fa6@0 & 6 & time & 202.65 & 2.47 \\
\hline misex1_Fd0@1 & 4 & 261.7 & 0.39 & 59.47 \\
\hline misex1_Fd1@0 & 4 & 60.7 & 0.47 & 149.73 \\
\hline misex1_Fd2@0 & 3 & 24.9 & 0.43 & 108.50 \\
\hline misex1_Fd3@1 & 3 & 24.0 & 0.26 & 72.07 \\
\hline misex1_Fy@0 & 5 & 16.5 & 0.17 & 11.12 \\
\hline misex1_Fy@1 & 5 & 15.2 & 0.30 & 28.15 \\
\hline misex3_Fa@0 & 9 & time & mem. & time \\
\hline misex3_Fa@1 & 9 & time & mem. & time \\
\hline misex3_Fb@0 & 9 & time & mem. & time \\
\hline misex3_Fb@1 & 8 & time & mem. & time \\
\hline pcler8_Fi@0 & 2 & 12.1 & 2.58 & 1.17 \\
\hline pcler8_Fi@1 & 2 & 19.8 & 2.37 & 3.52 \\
\hline pcler8_Fj@1 & 4 & 9.3 & 0.39 & 1.10 \\
\hline pcler8_Fk@1 & 4 & 8.2 & 0.28 & 5.48 \\
\hline term1_Fa@0 & 4 & 2.2 & 285.73 & 1.20 \\
\hline term1_Fb@0 & 7 & 513.2 & mem. & 27.63 \\
\hline term1_Fb@1 & 7 & 404.6 & 256.42 & 22.83 \\
\hline term1_Fc@0 & 4 & 75.4 & 0.86 & 9.95 \\
\hline term1_Fd@1 & 4 & 150.3 & 1.50 & 11.82 \\
\hline
\end{tabular}

Table 4. Algorithm comparison

A key aspect of the proposed techniques is the identification of a small set of dependencies explaining each identified conflict. In each case the main goal is to minimize the size of this set of dependencies, while guaranteeing that the resulting set still provides a sufficient explanation for the given conflict to occur. Future research work will naturally include seeking further simplification of the clauses created for each type of conflict. Moreover, additional techniques from the SAT domain can potentially be applied to solving BCP. These techniques are likely to be significant for instances of covering problems with sets of constraints that are hard to satisfy.

\section{References}

[1] P. Barth. A Davis-Putnam Enumeration Algorithm for Linear Pseudo-Boolean Optimization. Technical Report MPI-I-95-2-003, Max Plank Institute for Computer Science, 1995.

[2] O. Coudert. Two-Level Logic Minimization, An Overview. Integration, The VLSI Journal, vol. 17(2):677-691, October 1993.

[3] O. Coudert. On Solving Covering Problems. In Proceedings of the ACM/IEEE Design Automation Conference, June 1996.

[4] O. Coudert and J. C. Madre. New Ideas for Solving Covering Problems. In Proceedings of the ACM/IEEE Design Automation Conference, June 1995.

[5] M. Davis and H. Putnam. A Computing Procedure for Quantification Theory. Journal of the Association for Computing Machinery, vol. 7:201-215, 1960

[6] P. F. Flores, H. C. Neto, and J. P. Marques Silva. An exact solution to the minimum-size test pattern problem. In Proceedings of the IEEE International Conference on Computer Design, pages 510-515, October 1998 .

\begin{tabular}{|r|r|r|r|r|}
\hline \multicolumn{2}{|c|}{} & opbdp & min-prime & bsolo \\
\hline Benchmark & min. & CPU & CPU & CPU \\
\hline cordic_Fa2@0 & 6 & 2.78 & 0.09 & 0.21 \\
\hline cordic_Fa2@1 & 6 & 5.86 & 0.81 & 0.24 \\
\hline cordic_Fa3@0 & 6 & 5.99 & 0.09 & 0.18 \\
\hline cordic_Fa3@1 & 6 & 3.95 & 0.83 & 0.27 \\
\hline cordic_Fa4@1 & 6 & 2.27 & 0.63 & 0.18 \\
\hline cordic_Fa6@0 & 6 & 9.02 & 0.06 & 0.20 \\
\hline misex1_Fd0@1 & 4 & 0.06 & 0.14 & 0.25 \\
\hline misex1_Fd1@0 & 4 & 0.06 & 0.19 & 0.18 \\
\hline misex1_Fd2@0 & 3 & 0.06 & 0.09 & 0.23 \\
\hline misex1_Fd3@1 & 3 & 0.06 & 0.14 & 0.28 \\
\hline misex1_Fy@0 & 5 & 0.05 & 0.07 & 0.03 \\
\hline misex1_Fy@1 & 5 & 0.05 & 0.06 & 0.04 \\
\hline misex3_Fa@0 & 9 & 180.42 & 85.70 & 52.02 \\
\hline misex3_Fa@1 & 9 & 207.81 & 111.24 & 30.58 \\
\hline misex3_Fb@0 & 9 & 1354.45 & 549.04 & 95.83 \\
\hline misex3_Fb@1 & 8 & 987.35 & 394.73 & 79.69 \\
\hline pcler8_Fi@0 & 2 & 0.87 & 1.23 & 0.31 \\
\hline pcler8_Fi@1 & 2 & 0.74 & 0.40 & 0.39 \\
\hline pcler8_Fj@1 & 4 & 0.32 & 0.18 & 0.21 \\
\hline pcler8_Fk@1 & 4 & 0.48 & 0.28 & 0.51 \\
\hline term1_Fa@0 & 4 & 0.19 & 0.27 & 0.12 \\
\hline term1_Fb@0 & 7 & 4.29 & 1.10 & 0.31 \\
\hline term1_Fb@1 & 7 & 8.85 & 0.62 & 0.27 \\
\hline term1_Fc@0 & 4 & 38.59 & 1.67 & 0.29 \\
\hline term1_Fd@1 & 4 & 12.14 & 1.93 & 0.32 \\
\hline
\end{tabular}

Table 5. Algorithm comparison

[7] J. Gimpel. A Reduction Technique for Prime Implicant Tables. IEEE Transactions on Electronic Computers, vol. EC-14:535-541, August 1965.

[8] E. Goldberg, L. Carloni, T. Villa, R. K. Brayton, and A. L. Sangiovanni-Vincentelli. Negative thinking by incremental problem solving: application to unate covering. In Proceedings of the ACM/IEEE International Conference on Computer-Aided Design, 1997.

[9] G. Hachtel and F. Somenzi. Logic Synthesis and Verification Algorithms. Kluwer Academic Pub., 1996.

[10] S. Liao and S. Devadas. Solving Covering Problems Using LPRBased Lower Bounds. In Proceedings of the ACM/IEEE Design Automation Conference, 1997.

[11] V. M. Manquinho, P. F. Flores, J. P. Marques Silva, and A. L. Oliveira. Prime implicant computation using satisfiability algorithms. In Proceedings of the IEEE International Conference on Tools with Artificial Intelligence, pages 232-239, November 1997.

[12] D. De Micheli. Synthesis and Optimization of Digital Circuits. McGraw-Hill, 1994.

[13] G. L. Nemhauser and L. A. Wosley. Integer and Combinatorial Optimization. John Wiley \& Sons, 1988.

[14] J. P. Marques Silva and K. A. Sakallah. GRASP: A new search algorithm for satisfiability. In Proceedings of the ACM/IEEE International Conference on Computer-Aided Design, pages 220-227, November 1996.

[15] T. Villa, T. Kam, R. K. Brayton, and A. L. Sangiovanni-Vincentelli. Explicit and Implicit Algorithms for Binate Covering Problems. IEEE Transactions on Computer Aided Design, vol. 16(7):677-691, July 1997.

[16] S. Yang. Logic Synthesis and Optimization Benchmarks User Guide. Microelectronics Center of North Carolina, January 1991. 